\title{
Charmonium suppression by gluon bremsstrahlung in p-A and A-B collisions
}

\author{
J. Hüfner, Y.B. He \\ Institut für Theoretische Physik, Universität Heidelberg, \\ Philosophenweg 19, D-69120 Heidelberg, Germany \\ B.Z. Kopeliovich \\ Max-Planck Institut für Kernphysik, Postfach 103980, 69029 Heidelberg, Germany \\ Joint Institute for Nuclear Research, Dubna, 141980 Moscow Region, Russia
}

\begin{abstract}
Prompt gluons are an additional source for charmonium suppression in nuclear collisions, in particular for nucleus-nucleus collisions. These gluons are radiated as bremsstrahlung in $\mathrm{N}-\mathrm{N}$ collisions and interact inelastically with the charmonium states while the nuclei still overlap. The spectra and mean number $\left\langle n_{g}\right\rangle$ of the prompt gluons are calculated perturbatively and the inelastic cross section $\sigma_{\text {abs }}^{\Psi g}$ is estimated. The integrated cross sections $\sigma(A B \longrightarrow J / \psi X)$ for $\mathrm{p}-\mathrm{A}$ and $\mathrm{A}-\mathrm{B}$ collisions and the dependence on transverse energy for $\mathrm{S}-\mathrm{U}$ and $\mathrm{Pb}-\mathrm{Pb}$ can be described quantitatively with some adjustment of one parameter $\left\langle n_{g}\right\rangle \sigma_{\mathrm{abs}}^{\Psi g}$.
\end{abstract}

\section{INTRODUCTION}

The original proposal [1] of using charmonium production in heavy-ion collisions as a signal of QGP formation has triggered a series of experimental measurements at the CERN SPS in proton-nucleus and nucleus-nucleus reactions(c.f. the reviews [2, 3]). One remarkable 
observation out of these experiments [4 6] is: when one scans through data from the lighter to the heavier interacting nuclei, one sees that the $J / \psi$ suppression in $\mathrm{p}$-p up to central $\mathrm{S}-\mathrm{U}$ reactions is consistent with a picture of pure final-state absorption on nucleons, while the data for central $\mathrm{Pb}-\mathrm{Pb}$ collisions deviate from this systematics and are therefore termed "anomalous".

Among the many theoretical concepts we mention the following three mechanisms for $\Psi$ suppression which have been proposed (here $\Psi$ stands for $J / \psi, \chi_{c}, \psi^{\prime}$ ):

1. Inelastic collisions of a charmonium premeson $c \bar{c}$ by the nucleons of projectile and target. The inelastic cross section $\sigma_{\mathrm{abs}}^{c \bar{c} \Psi}(\tau)$ is a function of the time $\tau$ (measured in the rest system of the premeson until the asymptotic meson is formed) [7 [10. In most calculations an absorption cross section with adjusted value of $\sigma_{\text {abs }}=6 \mathrm{mb}$, independent of time, is used both for the $J / \psi$ and $\psi^{\prime}$ channel. While this approach reproduces the suppression in $\mathrm{p}-\mathrm{A}$ collisions and A-B collisions with small projectiles, central $\mathrm{Pb}-\mathrm{Pb}$ collisions show stronger suppressions.

2. Charmonium dissociation in the quark-gluon plasma phase of the reaction. The interaction of charmonium with the partons, in particular the gluons leads to suppression. The different binding energies of the states $J / \psi, \chi_{c}$, and $\psi^{\prime}$ play a crucial role and may lead to discontinuous behaviour in the $E_{T}$ dependence of the suppression [11 14].

3. In the final state of a nucleus-nucleus collision many hadrons emerge from the reaction volume together with the charmonium. The interaction with these comoving hadrons is an additional source of $\Psi$ suppression 15 18. In approaches of this kind, the inelastic cross section $\sigma_{\text {co }}^{\Psi}$ of the $\Psi$ with the comovers is the crucial adjusted parameter for which no reliable calculations are available at present.

In this situation we propose still another mechanism, suppression by prompt gluons. These gluons are produced as bremsstrahlung in the N-N collisions in the early phase of the reaction. Those gluons, whose production time is sufficiently short, will interact inelastically 
with the charmonium in addition to and at the same time as the $\Psi-\mathrm{N}$ interactions. In another language: The gluons produced in a N-N collision, $N+N \longrightarrow g+N+N$ may be seen together with $\mathrm{N}$ as a wounded nucleon $N^{*}=N+g$. In this language a $\Psi$ will be suppressed by collisions with nucleons $N$ in their ground state and by wounded nucleons, $N^{*}$. Whatever picture one prefers, the prompt gluons will be an additional source of suppression. Their number and their momentum distribution can be calculated perturbatively. Uncertainties remain for the inelastic cross section $\sigma_{\text {abs }}^{\Psi g}$.

In this paper we expand on the preliminary calculations which were presented in Ref. [19]. While the general idea is the same, calculations of the $\Psi$ suppression by nucleons and prompt gluons only, several improvements have been introduced and more observables have been calculated:

(i) A closed expression is derived within a classical multiple scattering model with straight line trajectories.

(ii) Formation time effects are included for the produced premeson and production time effects for the gluon by introducing a time dependence for the inelastic cross sections $\sigma_{\text {abs }}^{\Psi N}(t)$, $\sigma_{\text {abs }}^{\Psi g}(t)$ and for the mean numbers $\left\langle n_{g}\right\rangle(t)$ of prompt gluons.

(ii) We also calculate charmonium suppression in its dependence on transverse energy;

(iv) We predict suppressions for reactions with inverse kinematics.

\section{FORMALISM}

We follow the original idea of Ref. [19 and derive a closed expressions for charmonium suppression caused by nucleons and prompt gluons. The derivation is based on Fig.1, which displays a collision between a projectile nucleus $\mathrm{A}$ and a target nucleus $\mathrm{B}$ in the twodimensional $t-z$ (time-longitudinal coordinate) plane and in the NN c.m.s.. Since projectile and target nucleons have high energies (for the $200 \mathrm{GeV} / \mathrm{A}$ energy at SPS, projectile and target are characterized by a Lorentz-factor $\gamma=10$ ), the nucleons move nearly on the light-cone. Assuming zero production time for the premeson [10], a premeson is produced 
at point $O\left(t_{0}, z_{0}\right)$ and moves with a velocity $v_{c m}$ in the c.m.s.. During its motion along a straight line trajectory its internal structure develops in time towards the asymptotically observed $\Psi$. This evolution, called formation, is characterized by a certain formation time and manifests itself in a time dependent absorption cross section $\sigma_{\text {abs }}^{\Psi N}(t)$. It interacts at point $P\left(t_{1}, z_{1}\right)$ with a nucleon from the target $\mathrm{B}$. Before this encounter, this nucleon has experienced a collision at $Q\left(t_{2}, z_{2}\right)$ with another nucleon from the projectile, leading to the radiation of a prompt gluon (dashed line) which also interacts with the $\Psi$ at $P\left(t_{1}, z_{1}\right)$. The time difference $\Delta t_{\Psi}=t_{1}-t_{0}$ (measured in the NN c.m.s.) has to be put into relation to the formation time of the charmonium. Similarly the time difference $\Delta t_{g}=t_{1}-t_{2}$ has to be compared to the time it takes to produce a gluon (coherence time), which manifests itself in a time dependent mean number of gluons $\left\langle n_{g}\left(\Delta t_{g}\right)\right\rangle$. At the point $R\left(t_{3}, z_{3}\right)$ the $\Psi$ interacts with a projectile nucleon $N_{A}$ and its comoving prompt gluons.

First we treat the interaction of the premeson with target nucleon. The velocity of nucleons is taken as 1 (in unit of $c$ ). After created at point $O\left(t_{0}, z_{0}\right)$ but before colliding with the target nucleon $N_{B}$ at point $P\left(t_{1}, z_{1}\right)$, the premeson $\Psi$ has lived for a time interval $\Delta t_{\Psi}=t_{1}-t_{0}=\left(z-z_{0}\right) /\left(1+v_{c m}\right)$. Therefore, the contribution to $\Psi$ suppression from the target nucleons can be taken into account by an attenuation factor $e^{-X}$ with 


\section{FIGURES}

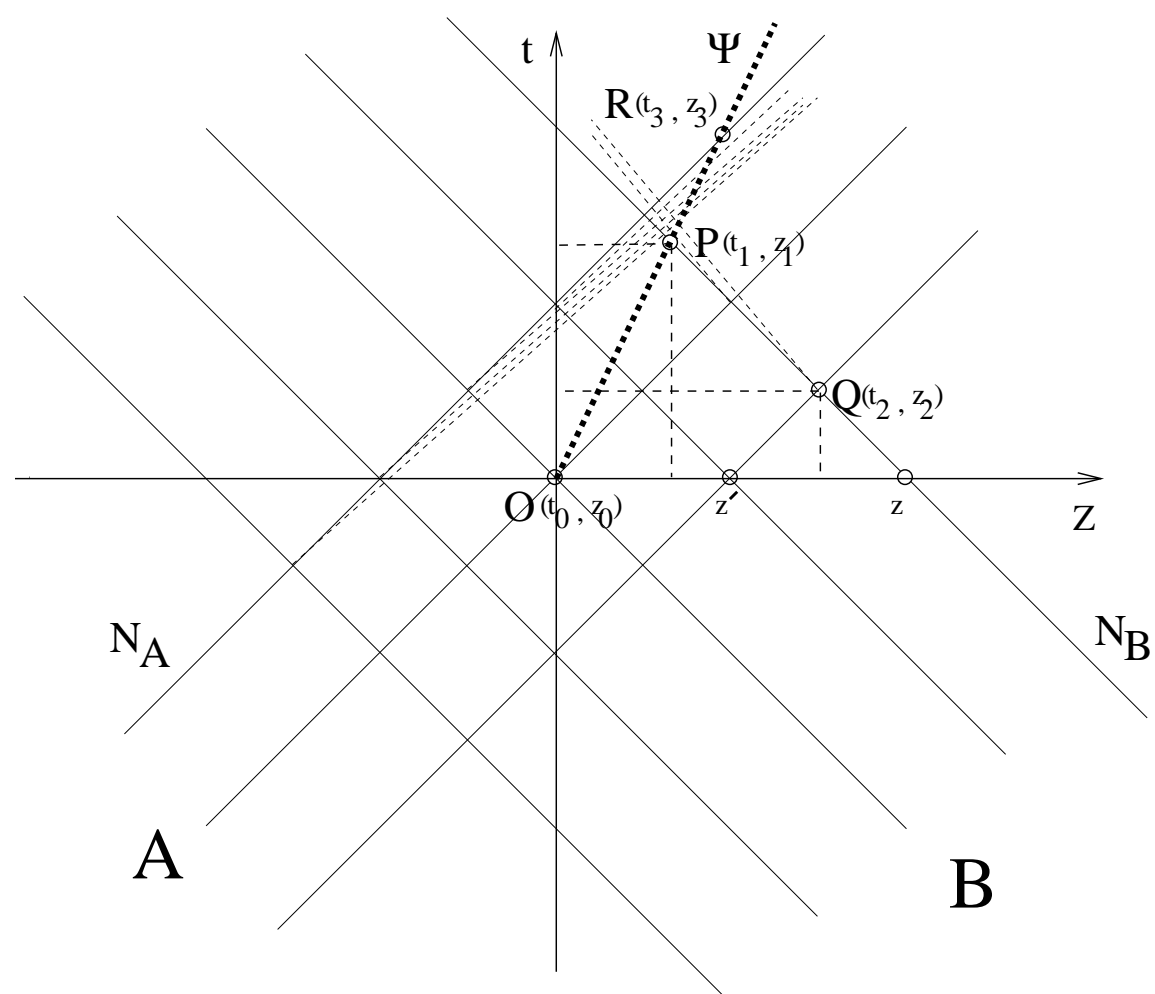

FIG. 1. The collision between a projectile nucleus A with a target nucleus B in the two dimensional time-longitudinal coordinate representation in the NN c.m. system. Nucleons are denoted by solid lines, the premeson produced at $O\left(t_{0,} z_{0}\right)$ is denoted by heavy dots, while the bremsstrahl gluons are drawn by light dashes.

$$
X=\int_{z_{0}}^{\infty} d z \gamma \rho_{B}(z) \sigma_{\mathrm{abs}}^{\Psi N}\left(\Delta t_{\Psi}\right)
$$

where we have suppressed the impact parameter dependence of $\rho_{B}$, the density of the target

nucleus B. The time dependent inelastic cross section $\sigma_{\mathrm{abs}}^{\Psi N}\left(\Delta t_{\Psi}\right)$ accounts for the formation of the charmonium state (see below).

We treat the interaction with prompt gluons in the following way: The target nucleon $N_{B}$ collides with a projectile nucleon at point $Q\left(t_{2}, z_{2}\right)$ and a gluon is radiated. This radiation process is not instantaneous, but is governed by a characteristic time, the production or coherence time (see below). The gluon radiated at $Q\left(t_{2}, z_{2}\right)$ interacts with the $\Psi$ at the point $P\left(t_{1}, z_{1}\right)$ after a time interval,

$$
\Delta t_{g}=\Delta t_{\Psi}-\frac{z-z^{\prime}}{2}
$$


The contribution to $\Psi$ suppression from gluons is therefore given by an attenuation factor $e^{-Y}$ with

$$
Y=\int_{z_{0}}^{\infty} d z \gamma \rho_{B}(z) \sigma_{\mathrm{abs}}^{\Psi g}\left(\Delta t_{\Psi}\right) \int_{-\infty}^{+\infty} d z^{\prime} \Theta\left(\Delta t_{g}\right) \gamma \rho_{A}\left(z^{\prime}\right) \sigma_{\mathrm{in}}^{N N}\left\langle n_{g}\left(\Delta t_{g}\right)\right\rangle
$$

where $\sigma_{\text {in }}^{N N}$ is the inelastic N-N cross section, $\left\langle n_{g}\left(\Delta t_{g}\right)\right\rangle$ the mean number of gluons radiated in a $\mathrm{N}-\mathrm{N}$ interaction (to be discussed below), and the $\Theta$-function ensures that only gluons, which are created before the interaction with the $\Psi$, are taken into account.

The suppression of the $\Psi$ via the interaction with projectile nucleons and their accompanying gluons can be derived by appropriate variable exchanges. Combining these results together one can write down the $\Psi$ suppression factor in $A-B$ collisions as:

$$
S_{A B}^{\Psi}=\frac{1}{A B} \int d^{2} b \int d^{2} s \int_{-\infty}^{+\infty} d z_{A} \gamma \int_{-\infty}^{+\infty} d z_{B} \gamma \rho_{A}\left(z_{A}, \vec{s}\right) \rho_{B}\left(z_{B}, \vec{b}-\vec{s}\right) \exp \left[-I_{1}-I_{2}\right]
$$

where $I_{1}$ is given by

$$
\begin{aligned}
I_{1}= & \int_{z_{A}}^{+\infty} d z \gamma \rho_{A}(z, \vec{s}) \sigma_{\mathrm{abs}}^{\Psi N}\left(\Delta t_{\Psi}\right) \\
& \times\left[1+\frac{\sigma_{\mathrm{abs}}^{\Psi g}}{\sigma_{\mathrm{abs}}^{\Psi N}} \int_{-\infty}^{+\infty} d z^{\prime} \gamma \Theta\left(\Delta t_{g}\right) \rho_{B}\left(z^{\prime}, \vec{b}-\vec{s}\right) \sigma_{\mathrm{in}}^{N N}\left\langle n_{g}\left(\Delta t_{g}\right)\right\rangle\right],
\end{aligned}
$$

and $I_{2}$ is derived from $I_{1}$ by the substitutions: $z_{A} \rightarrow z_{B}, \rho_{A}(z, \vec{s}) \rightarrow \rho_{B}(z, \vec{b}-\vec{s}), \rho_{B}\left(z^{\prime}, \vec{b}-\right.$ $\vec{s}) \rightarrow \rho_{A}\left(z^{\prime}, \vec{s}\right), v_{c m} \rightarrow-v_{c m}$ (hidden in $\left.\Delta t_{\Psi}\right)$.

The first and second terms in the exponential in Eq. (3) correspond to contributions from the nuclei $\mathrm{A}$ and $\mathrm{B}$, respectively. In the absence of prompt gluons, $\left\langle n_{g}\right\rangle=0$, and neglecting the dependence on $\Delta t_{\Psi}$, one has the usual expression for charmonium suppression by nucleons. The $E_{T}$ dependence of the charmonium suppression is calculated from the impact parameter dependent suppression function

$$
S_{A B}^{\Psi}(\vec{b})=\frac{\int d^{2} s \int_{-\infty}^{+\infty} d z_{A} \gamma \int_{-\infty}^{+\infty} d z_{B} \gamma \rho_{A}\left(z_{A}, \vec{s}\right) \rho_{B}\left(z_{B}, \vec{b}-\vec{s}\right) \exp \left[-I_{1}-I_{2}\right]}{\int d^{2} s \int_{-\infty}^{+\infty} d z_{A} \gamma \int_{-\infty}^{+\infty} d z_{B} \gamma \rho_{A}\left(z_{A}, \vec{s}\right) \rho_{B}\left(z_{B}, \vec{b}-\vec{s}\right)}
$$

The suppression for a given value $E_{T}$ is then calculated from

$$
S_{A B}^{\Psi}\left(E_{T}\right)=\int d^{2} b P\left(\vec{b} ; E_{T}\right) S_{A B}^{\Psi}(\vec{b})
$$


where the distribution $d^{2} b P\left(\vec{b} ; E_{T}\right)$ gives the probability that a given impact parameter contributes to a particular value of $E_{T}$. We use the following simplification: The NA50/51 group has made simulations and give the mean value $\langle b\rangle\left(E_{T}\right)$ for each values of $E_{T}$ where data exist. We have inserted these values into Eq. (5).

Proton-nucleus collisions can be treated as a special case of Eq. (3).

For the time dependent effective cross section $\sigma_{\text {abs }}^{\Psi N}\left(\Delta t_{\Psi}\right)$ between the charmonium state $\Psi$ and a nucleon the expression of the two-channel model for the evolution of a $c \bar{c}$ pair is used [20,10]:

$$
\left.\sigma_{\text {abs }}^{\Psi N}(\tau)=\sigma_{\text {in }}^{\Psi N}+\left(\sigma_{\text {in }}^{\text {pre }}-\sigma_{\text {in }}^{\Psi N}\right) \cos (\Delta M \tau)\right]
$$

where $\sigma_{\text {in }}^{\Psi N}$ is the asymptotic $(\tau \rightarrow \infty) \Psi-N$ cross section, $\sigma_{\text {in }}^{\text {pre }}$ the initial $(\tau \rightarrow 0)$ premeson$\mathrm{N}$ cross section, and $\Delta M=M_{\psi^{\prime}}-M_{J / \psi}$. We use values $\sigma_{\text {in }}^{J / \psi N}=6.7 \mathrm{mb}$ (which includes the contributions of $\chi_{c}$ and $\left.\psi^{\prime}\right)$ and $\sigma_{\text {in }}^{\psi^{\prime} N}=12 \mathrm{mb}$. For $\tau \rightarrow 0$ we have the absorption cross section of the premeson, $\sigma_{\text {in }}^{\text {pre }}$, which we take the same in the $J / \psi$ and $\psi^{\prime}$ channels to be 3 $\mathrm{mb}[10$.

The mean number $\left\langle n_{g}\right\rangle$ of gluons radiated in a $N-N$ collision in the direction of one of the two nucleons has been calculated in [19]:

$$
\left\langle n_{g}\left(t_{g}\right)\right\rangle=\frac{3}{\sigma_{i n}^{N N}} \int_{k_{\min }^{2}}^{\infty} d k^{2} \int_{\alpha_{\min }}^{1} d \alpha \frac{d \sigma(q N \rightarrow g X)}{d \alpha d k^{2}}\left[1-\exp \left(-\frac{t_{g}}{t_{c}^{g}}\right)\right]
$$

where 22

$$
\frac{d \sigma(q N \rightarrow g X)}{d \alpha d k^{2}}=\frac{3 \alpha_{s}\left(k^{2}\right) C}{\pi} \frac{2 m_{q}^{2} \alpha^{4} k^{2}+\left[1+(1-\alpha)^{2}\right]\left(k^{4}+\alpha^{4} m_{q}^{4}\right)}{\left(k^{2}+\alpha^{2} m_{q}^{2}\right)^{4}}\left[\alpha+\frac{9}{4} \frac{1-\alpha}{\alpha}\right] .
$$

Here $\alpha_{s}\left(k^{2}\right)$ is the QCD running coupling constant; $C$ is the factor for the dipole approximation for the cross section of a $\bar{q} q$ pair with a nucleon, $\sigma^{\bar{q} q}\left(r_{T}\right) \approx C r_{T}^{2}$, where $r_{T}$ is the $\bar{q} q$ transverse separation $[23,24,22] . C \approx 3$ is the pQCD prediction. The coherence time is given by

$$
t_{c}^{g}=\frac{2 E_{q} \alpha(1-\alpha)}{\alpha^{2} m_{q}^{2}+k^{2}}
$$


and the lower limit of the $\alpha$-integration

$$
\alpha_{\min }= \begin{cases}\left(\omega_{\min }+\sqrt{\omega_{\min }^{2}-k^{2}}\right) /\left(2 E_{q}\right) & \text { if } \mathrm{k}<\omega_{\min }, \\ k /\left(2 E_{q}\right) & \text { otherwise. }\end{cases}
$$

Here $\alpha$ is the fraction of the quark light-cone momentum and $k$ the transverse momentum carried by the gluon. Compared to Ref. [19] we have introduced a time dependence into the mean number of gluons by inserting the square bracket into the integral Eq. (7). While the limits $t \rightarrow 0$ and $t \rightarrow \infty$ and the characteristic time $t_{c}^{g}$ for the rise are certainly correct, the explicit form of the exponential is arbitrary. In our calculation we use $k \geq 0.5 \mathrm{GeV} / c$ and thus restrict ourselves to (semi-)hard gluons. Their energy $\omega \geq \omega_{\min }$ has to be chosen in relation to the type of charmonium. For example, the destruction of $J / \psi$ and $\psi^{\prime}$ needs $\omega_{\min }=0.7 \mathrm{GeV}$ and $0.1 \mathrm{GeV}$, respectively. However, as long as the premeson are still in formation, the uncertainty relation tells us that the binding energies are not yet the final ones.

The choice of $\omega_{\text {min }}$ deserves a special discussion since it is related to the problem of the energy dependence of $\Psi-g$ break-up cross section $\sigma_{\mathrm{abs}}^{\Psi g}$ in Eq. (雨). At high energies it is dominated by gluonic exchange in t-channel and slightly grows with energy [21]. The inelastic $\Psi-g$ cross section is by a color factor $9 / 4$ different from the $\Psi-q$ cross section, which is according to the constituent quark model related to the $\Psi$-N cross section. Therefore, one expects

$$
\sigma_{\mathrm{abs}}^{\Psi g} \simeq \frac{9}{4} \sigma_{\mathrm{abs}}^{\Psi q} \simeq \frac{3}{4} \sigma_{\mathrm{abs}}^{\Psi N}
$$




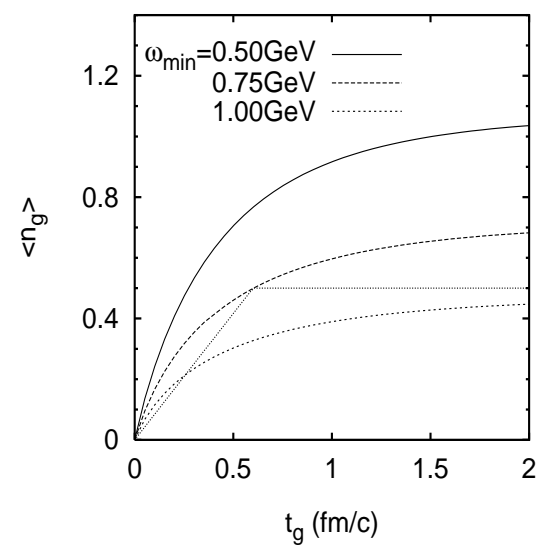

FIG. 2. Mean number of prompt gluons as a function of the time $t_{g}$ after the NN collision for various values of $\omega_{\min }$.

We are left with one adjustable parameter $\omega_{\min }$ in Eq. (7). In Fig. 2 we show the mean number of gluons, $\left\langle n_{g}\right\rangle$, as a function of length $t_{g}$ for different choices of parameter $\omega_{\min }$. In the numerical results presented in the next Section we will not use the form of Fig. 2 which is generated by the somewhat arbitrary exponential form in Eq. (7), but use a simplified form of $\left\langle n_{g}\left(t_{g}\right)\right\rangle$ in order to reduce the numerical efforts:

$$
\left\langle n_{g}\left(t_{g}\right)\right\rangle= \begin{cases}n_{g}^{0} t_{g} / t_{0} & \text { if } \mathrm{t}_{\mathrm{g}}<\mathrm{t}_{0}, \\ n_{g}^{0} & \text { if } \mathrm{t}_{\mathrm{g}} \geq \mathrm{t}_{0} .\end{cases}
$$

This step function type behaviour is also shown in Fig. 2. In our calculations we have used a fixed value $t_{0}=0.6 \mathrm{fm} / c$, and have varied $n_{g}^{0}$ within certain limits, $0.5 \leq n_{g}^{0} \leq 1$ for the $J / \psi$ (corresponding to $\omega_{\min }$ between 0.5 and $1 \mathrm{GeV}$ ) and $1 \leq n_{g}^{0} \leq 2$ for the $\psi^{\prime}$ because of its smaller binding energy. 


\section{RESULTS AND DISCUSSIONS}

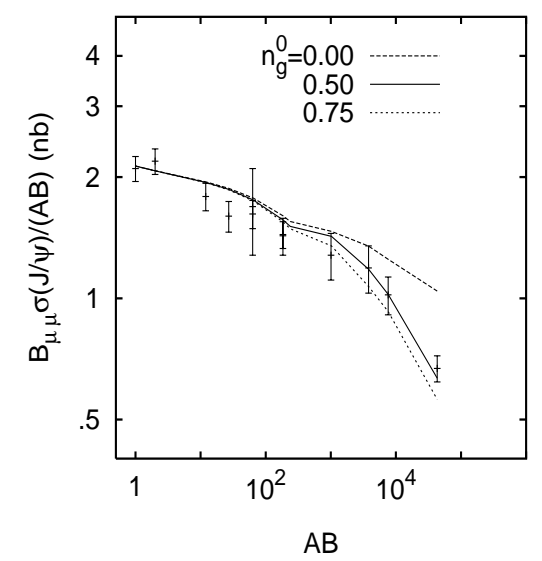

FIG. 3. The $J / \psi$ total cross section as a function of the product $A B$ of the projectile and target atomic mass numbers at $200 \mathrm{GeV} / \mathrm{c}$, for $n_{g}^{0}=0,0.5,0.75$. The data are from 25].

We have calculated the total cross section of $J / \psi$ production in proton-nucleus and nucleus-nucleus reactions according to Eq. (3), and the results are shown in Fig. 3 for three different values of $n_{g}^{0}$. The absolute values of cross section are normalized to the mean value of those in $\mathrm{p}-\mathrm{p}$ and $\mathrm{p}-\mathrm{d}$ reactions $\mathrm{AB}=1$ and 2 . The long-dashed curve corresponds to the result when there are no gluons (only suppression due to nucleons), and cannot reproduce the experimental data for the $\mathrm{Pb}-\mathrm{Pb}$ reactions, while the solid line with $n_{g}^{0}=0.5$ accounts for all the data very well.

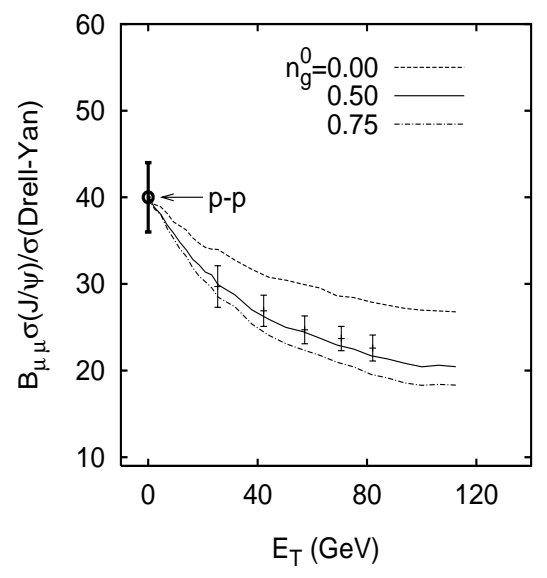


FIG. 4. Ratio of $J / \psi$ to Drell-Yan cross section as a function of centrality in S-U collisions at $200 \mathrm{GeV} / \mathrm{c}$, with $n_{g}^{0}=0,0.5,0.75$. The data points are from [6]. The calculated curves are normalized at $E_{T}=0$ to the ratio observed for p-p collisions.

Various experiments measure charmonium production as a function of the transverse energy $E_{T}$ which corresponds to different centralities. Fig. 1 shows the calculated suppression in $\mathrm{S}-\mathrm{U}$ collisions as a function of $E_{T}$ with the normalization at $E_{T}=0$ (p-p data). Note that the rather large uncertainty introduced by this normalization.

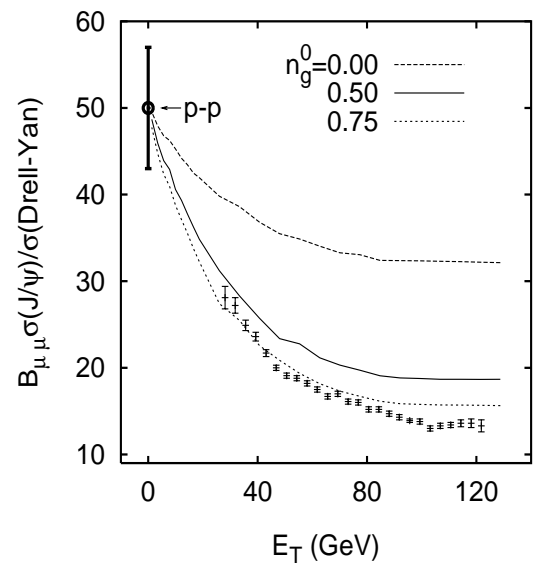

FIG. 5. Ratio of $J / \psi$ to Drell-Yan cross section as a function of centrality in $\mathrm{Pb}-\mathrm{Pb}$ collisions at $158 \mathrm{GeV} / \mathrm{c}$ for $n_{g}^{0}=0,0.5,0.75$. The data are from [6]. The calculated curves are normalized at $E_{T}=0$ to the ratio observed for p-p collisions.

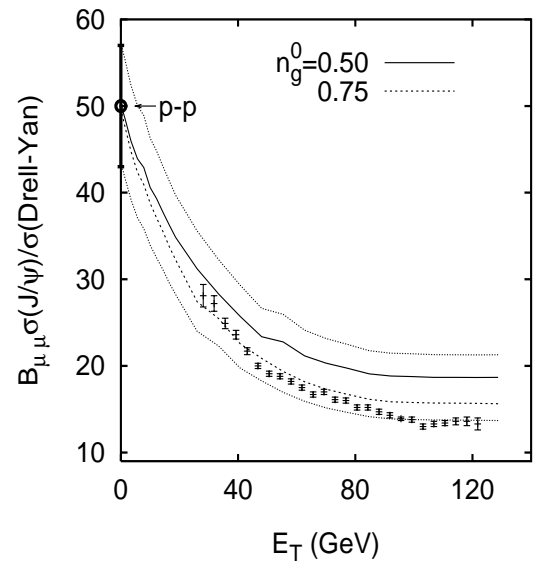


FIG. 6. Ratios of $J / \psi$ to Drell-Yan cross section as a function of centrality in $\mathrm{Pb}-\mathrm{Pb}$ collisions at $158 \mathrm{GeV} / \mathrm{c}$ for $n_{g}^{0}=0.5,0.75$ (the two curves in the middle). The upper curve corresponds to the result for $n_{g}^{0}=0.5$ but normalized at $E_{T}=0$ to p-p data point with a standard deviation higher, and the lower one is the $n_{g}^{0}=0.75$ curve normalized at $E_{T}=0$ to p-p data point with a standard deviation lower.

A comparison of our calculation with NA50 data for $\mathrm{Pb}-\mathrm{Pb}$ collisions at $158 \mathrm{GeV} / \mathrm{c}$ is presented in Fig. 5 for $n_{g}^{0}=0,0.5,0.75$. As for $\mathrm{S}-\mathrm{U}$ the calculation is normalized at $E_{T}=0$ to the p-p point. It is obvious that a theory with no gluons cannot reproduce the data. The value $n_{g}^{0}=0.5$ which is predicted for $\omega_{\min }=0.75$ (which corresponds to the $\mathrm{J} / \psi$ binding energy) fits the absolute $J / \psi$ production cross sections (Fig. 3) well, but seems a little high in the $E_{T}$ distribution for $\mathrm{Pb}-\mathrm{Pb}$ collisions. However, one also has to take into account that there is a nearly $15 \%$ uncertainty in the normalization (see Fig. 6).

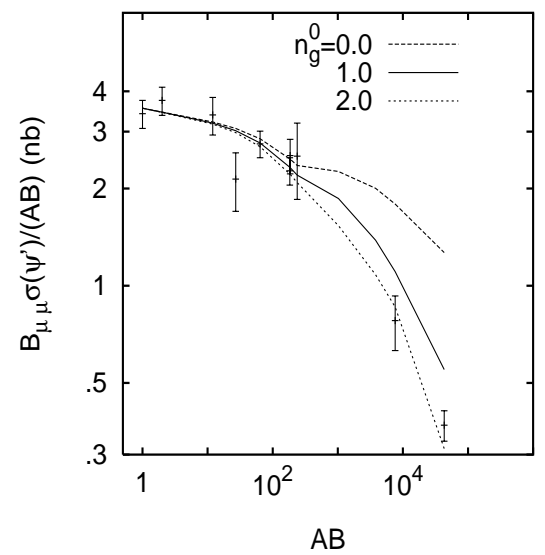

FIG. 7. The integrated $\psi^{\prime}$ production cross section as a function of the product $A B$ of the projectile and target atomic mass numbers at $200 \mathrm{GeV} / \mathrm{c}$. 


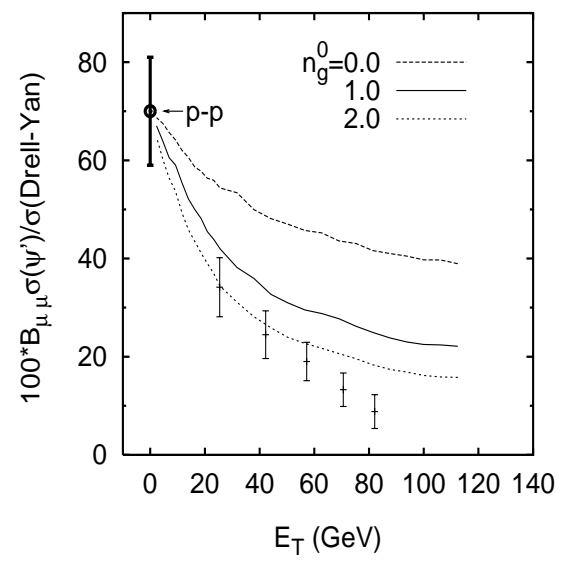

FIG. 8. The $\psi^{\prime}$ cross section relative to the Drell-Yan cross section as a function of the transverse energy for S-U collisions at $200 \mathrm{GeV} / \mathrm{c}$ [5].

We proceed to the suppression of $\psi^{\prime}$ in nuclear collisions. Since the $\psi^{\prime}$ is less bound than the $J / \psi$, we may take $\omega_{\min } \geq 200 \mathrm{GeV}$ and correspondingly $n_{g}^{0} \simeq 1-2$ is the theoretical choice and leads to reasonable description of the absolute production cross section (Fig. 7), but the $E_{T}$ distribution in $\mathrm{S}-\mathrm{U}$ is not well reproduced (Fig.8). The data seem to fall faster than the calculation.

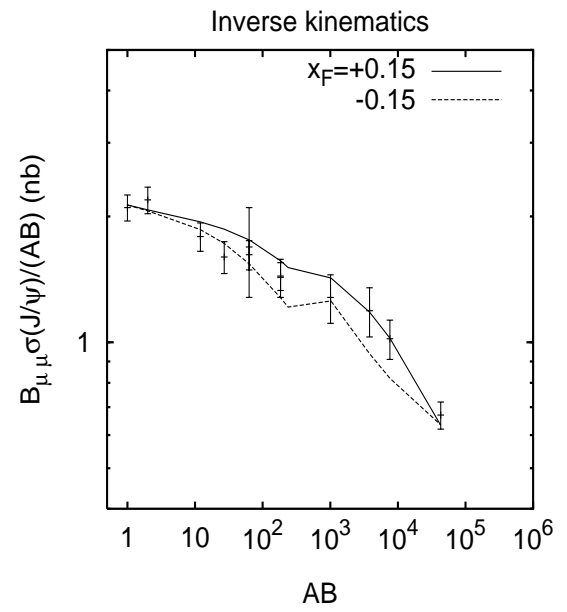

FIG. 9. The integrated $J / \psi$ total cross sections in nuclear collisions in the experimental configuration $\left(x_{F}=0.15\right)$ and in the situation of inverse kinematics $\left(x_{F}=-0.15\right)$. 


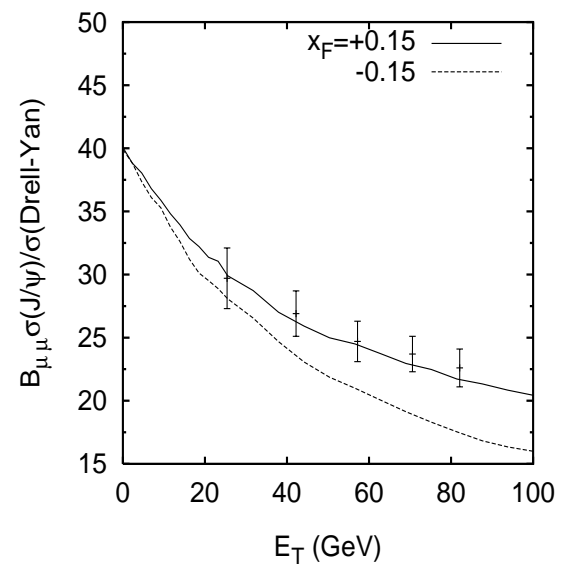

FIG. 10. Transverse energy distribution of $J / \psi$ production relative to the Drell-Yan for S-U collisions $\left(x_{F}=0.15\right)$ and hypothetical U-S collisions $\left(x_{F}=-0.15\right)$.

Finally, we have calculated the integrated and $E_{T}$ dependent cross sections of $J / \psi$ production for the inverse kinematics, i.e. p-A and A-p collisions and S-U and U-S collisions. We expect differences, since the charmonium is observed with a finite rapidity with respect to the NN c.m. system. In our calculation we just exchange $v_{c m} \rightarrow-v_{c m}$ of the charmonium. The differences arise because of the formation and production times. Figs. 9 and 10 show two results. The effects of inverse kinematics are significant but not dramatic.

\section{SUMMARY AND CONCLUSIONS}

We have calculated charmonium suppression in nuclear collisions for $J / \psi$ and $\psi^{\prime}$ and for integrated cross sections and differential ones with respect to transverse energy.

The emphasis of the present paper has been on the contribution of the prompt gluons to the suppression. This contribution turns out to be significant and decisive, and can quantitatively account for the suppressions in almost all cases. However, since the values of the input variables $n_{g}^{0}$ and $\sigma^{\Psi g}$ bear considerable uncertainty, we cannot exclude that other mechanisms (plasma, hadronic comovers) will also contribute. In these calculations several parameters are adjusted so that the relative importance of the various contributions, including those of prompt gluons, cannot be quantitatively determined at the moment. 


\section{ACKNOWLEDGMENTS}

We thank C. Gerschel and A. Mueller for several comments. This paper has been supported in part by a grant from the BMBF under contract number 06 HD 742. 


\section{REFERENCES}

[1] T. Matsui and H. Satz, Phys. Lett. B178 (1986) 416.

[2] R. Vogt, Phys. Rep. 310 (199) 197.

[3] C. Gerschel and J. Hüfner, Ann. Rev. Nucl. Part. Scie in print, 1999.

[4] M.C. Abreu et al. (NA51 Coll.), Phys. Lett. B438 (1998) 35.

[5] M.C. Abreu et al. (NA38 Coll.), Phys. Lett. B444 (1998) 516.

[6] M.C. Abreu et al., Phys. Lett. B449 (1999) 128.

[7] S.J. Brodsky and A.H. Mueller, Phys. Lett. B206 (1988) 685.

[8] B.Z. Kopeliovich and B.G. Zakharov, Phys. Rev. D 44, 3466 (1991).

[9] F. Arleo, P.-B. Gossiaux, T. Gousset, and J. Aichelin, Charmonium suppression in p-A collisions, hep-ph/9907286.

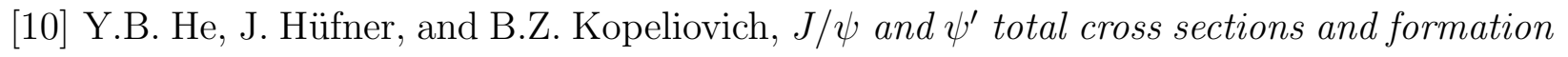
times from data for charmonium suppression in $p$ A collisions, hep-ph/?.

[11] J.-P. Blaizot and J.-Y. Ollitrault, Phys. Rev. Lett. 77, 1703 (1996).

[12] C.-Y. Wong, Phys. Rev. C 55, 2621 (1997).

[13] D. Kharzeev, C. Lourenço, M. Nardi and H. Satz, Z. Phys. C74, 307 (1997).

[14] R. Vogt, Phys. Lett. B430 (1998) 15.

[15] S. Gavin and R. Vogt, Phys. Rev. Lett. 78, 1006 (1997).

[16] A. Capella, A.B. Kaidalov, A. Kouider Akil and C. Gerschel, Phys. Lett. B393 (1997) 431.

[17] N. Armesto, A. Capella and E.G. Ferreiro, Phys. Rev. C 59, 395 (1999). 
[18] W. Cassing and C.M. Ko, Phys. Lett. B396 (1997) 39; J. Geiss, C. Greiner, E.L. Bratkovskaya, W. Cassing and U. Mosel, Phys. Lett. B447 (1999) 31.

[19] J. Hüfner and B.Z. Kopeliovich, Phys. Lett. B445 (1998) 223.

[20] J. Hüfner and B.Z. Kopeliovich, Phys. Rev. Lett. 76 (1996) 192.

[21] J. Hüfner and B.Z. Kopeliovich, Phys. Lett. B426 (1998) 154.

[22] B.Z. Kopeliovich, A. Schfer, A.V. Tarasov, Phys. Rev. C 59, 1609 (1999).

[23] A.B. Zamolodchikov, B.Z. Kopeliovich, L.I. Lapidus, JETP Lett. 33 (1981) 595.

[24] B. Povh and J. Hüfner, Phys. Rev. Lett. 58 (1987) 1612.

[25] M.C. Abreu et al., Phys. Lett. B410 (1997) 337.

[26] M.C. Abreu et al., Phys. Lett. B450 (1999) 456. 